\title{
SENSIBILIZAÇÃO SOBRE A PRÁTICA DA GINÁSTICA LABORAL (GL) PARA ADERÊNCIA A PRATICA DE ATIVIDADE FÍSICA FORA DO AMBIENTE DE TRABALHO.
}

\author{
Marcelo José Alves, Adriana Junqueira \\ Universidade do Oeste Paulista - UNOESTE, Curso de Educação Física, Presidente Prudente, SP. E-mail: \\ a.junqueira@unoeste.br
}

\begin{abstract}
RESUMO
Este trabalho observou se um trabalho de sensibilização iria promover a aderência à prática de atividade física fora do ambiente de trabalho, objetivando analisar se os praticantes de Ginástica Laboral (GL) participam ou já participaram de alguma atividade física fora de seu serviço; se através da sensibilização eles passaram a observar a importância que tem; qual estágio de aderência se encontram e se houve mudanças no decorrer do trabalho. Foi utilizado um questionário contendo informações pertinentes a prática da ginástica laboral e orientações através de um mural, sobre os benefícios da prática regular de atividade física. O grupo foi composto por 45 funcionários da instituição de ensino Unoeste, analisamos sua participação durante as aulas de ginástica laboral sensibilizando-os a aderir a pratica de atividade física, após 20 dias foi realizada uma reavaliação, concluímos que houve a sensibilização dos participantes.
\end{abstract}

Palavra-chave: Ginástica laboral. Sensibilização. Atividade Física. Aderência. Labor

\section{AWARENESS ABOUT THE PRACTICE OF LABOR GYMNASTICS (LB) FOR COHERE PRACTICE OF PHYSICAL ACTIVITY OUTSIDE THE WORKPLACE.}

\begin{abstract}
This study observed a sensitization work would promote cohere to physical activity outside of work environment, in order to analyze whether the practitioners of Labor Gymnastics (GL) participate or have participated in any physical activity outside of their service; if by raising awareness they began to notice the importance of; which adherence stage are and whether there were changes in the course of work. a questionnaire containing information relevant to the practice of gymnastics was used and guidance through a wall, on the benefits of regular physical activity. The group was composed of 45 employees Unoeste educational institution, we analyze participation during gymnastics classes sensitizing them to join the practice of physical activity, after 20 days the patient was revaluated, we concluded that there was awareness of the participants.
\end{abstract}

Keyword: Labor Gymnastics. Awareness. Physical. Cohere .Labor. 


\section{INTRODUÇÃO}

A Ginástica Laboral (GL) é a prática da atividade física orientada e dirigida durante o horário do expediente e no local de trabalho, isto é, existe uma pausa para que possam ser realizados exercícios físicos que visam benefícios pessoais e no trabalho ${ }^{1}$.

De acordo com Polito e Bergamashi ${ }^{2}$, nota-se o quanto é importante a adesão a prática da atividade física dentro do ambiente de trabalho. Quando se prática GL promovemos uma melhora física, mental e social ao individuo. Segundo Cantarino \& Pinheiro ${ }^{3}$, Monteiro ${ }^{4}$ e MGM $^{5}$, a ginástica laboral, além de prevenir as L.E.R. (Lesões por Esforço Repetitivo) e D.O.R.T. (Doenças Osteomusculares Relacionadas ao Trabalho), busca a eficiência e saúde musculoesquelético, redução dos riscos ambientais, redução do estresse e interesse conjunto da empresa e dos funcionários em melhorias de qualidade de vida.

Desta forma Bulsing ${ }^{6}$, acrescenta ainda que com a prática de GL ocorre a liberação de movimentos bloqueados por tensões emocionais, obtendo a sensação de um corpo mais relaxado, melhora na coordenação motora dos funcionários, reduzindo, assim, o gasto de energia para a execução de suas tarefas. Neste sentido, é importante saber que a GL não é suficiente para combater doenças como obesidade, pressão alta, diabetes, entre outras patologias, é necessária a conscientização da importância de praticar alguma atividade física regular fora do ambiente de trabalho.

A GL auxilia na melhora física, social e psicológica dos participantes, combatendo e prevenindo a LER e DORT ${ }^{2}$. Segundo Albuquerque ${ }^{7}$, promove ainda diversos benefícios na vida dos trabalhadores que são divididos: Fisiológicos, Psicológicos e Sociais

As adaptações fisiológicas são estímulos para o aumento da temperatura corporal, tecidual e da circulação sanguínea durante o momento em que a região está sendo exercitada ${ }^{8}$. Baseado nisso Alter ${ }^{9}$, diz que o aquecimento pode ser dividido em: passivo e ativo. $O$ aquecimento passivo envolve agentes externos como: banhos quentes, luz infravermelha, etc.

Entende-se o papel que a GL tem no ambiente de trabalho como ferramenta promotora de conscientização para a aderência a alguma atividade física fora ambiente de trabalho. A prática regular de atividades físicas representa um passo importante para a mudança do modus vivendi (modo de viver) de uma pessoa. Trocar o elevador e as escadas rolantes pelas escadas convencionais, usar menos o carro, ou saltar do ônibus um ponto antes, preferindo a caminhada, são estratégias eficazes para tornar a rotina de um individuo mais ativa ${ }^{6}$.

De acordo com o ACSM (American College of Sports Medicine) ${ }^{10}$, juntamente com o centro de prevenção e controle de doenças, em cooperação com o conselho presidencial para o condicionamento físico e os esportes, a atividade física esta intimamente relacionada com o estilo de vida, pois segundo eles ambos são de grande importância para a melhora da qualidade de vida e saúde das pessoas. São descritos os seguintes benefícios da atividade física regular e/ou exercício físico: aprimoramento na função cardiovascular e respiratória; redução nos fatores de risco para doença arterial coronariana; mortalidade e morbidez reduzidas e outros benefícios tais como menor ansiedade e depressão; sensações de bem-estar aprimoradas e melhor desempenho nas atividades laborativas, recreativas e desportivas.

Observando os benefícios proporcionados pela AF (atividade física) segundo a $\mathrm{ACSM}^{10}$, é importante salientar sua influência positiva quando incluída no cotidiano, pois a pratica de alguma atividade, seja ela qual for que nos coloque em um estado esforço maior, estaremos melhorando nosso estado de saúde, equilibrando nossas funções orgânicas e ao mesmo tempo nos prevenindo doenças crônicas. Segundo Nahas ${ }^{11}$, é definida como sendo um conjunto de ações cotidianas que reflete as atitudes e valores das pessoas e por sua vez, pode mudar ao longo dos anos, mas isso só ocorrerá se a pessoa conscientemente enxergar algum motivo relevante para incluir ou excluir valores ou atitudes, assim como se sentir capaz de realizar as mudanças pretendidas. 
À medida que o individuo declina fisicamente pode haver deteriorização concomitante na sensação de bem-estar, resultando em autoestima precária, ansiedade, fadiga e depressão ${ }^{12}$. Esses estão frequentemente associados a pouca motivação e uma redução adicional na atividade física. Através de um modelo transteórico, segundo Prochasca \& Marcus ${ }^{13}$, prevê uma progressão no processo de aderência ao estilo de vida ativo através de cinco estágios:

- $\quad$ Pré-contemplação ou pré-expectativa: estagio em que o individuo não pratica e nem pretende se tornar uma pessoa ativa;

- $\quad$ Expectativa ou Contemplação: estagio onde o individuo ainda é sedentário, mas já começa a perceber a importância do exercício físico;

- Preparação: estagio onde o individuo começa a viabilizar uma pratica ocasional e sem regularidade para a prática do exercício físico;

- Ação: estagio onde o individuo concede a si uma oportunidade de experimentar o exercício físico. A participação do profissional de educação física nessa fase torna-se fundamental, pois é a fase de maior índice de desistência;

- Manutenção: estagio onde a pratica torna-se regular a mais de seis meses, aumentando a probabilidade do individuo se manter em atividade.

De forma simplificada, alguns autores traduzem os diferente estágios de aderência como: Eu não vou/pré contemplação; Eu talvez vá/ contemplação; Eu vou/ preparação; Eu estou/ ação; Eu tenho (... um habito saudável)/manutenção ${ }^{14}$.

Analisando motivos para que os indivíduos se conscientizem sobre à pratica de alguma atividade física, algumas razões foram apontadas para se exercitar ${ }^{15}$ :

- Controle do peso corporal: tendência da valorização social da magreza e preocupação com a aparência.

- Queda do risco de hipertensão: pesquisas evidenciam que exercícios regulares contribuem para a prevenção de doenças cardiovasculares, portanto, acontece à busca do indivíduo em virtude da informação obtida, ou por recomendação médica.

- Queda do estresse e da depressão: devido aos últimos anos terem aumentado os indivíduos com transtornos de ansiedade e depressão, as pessoas procuram atividade física também por obtenção de informação ou recomendação médica para prevenção ou tratamento de ambos.

- Satisfação: este aspecto é mais ligado à manutenção. $O$ indivíduo precisa divertir-se ao executar a atividade.

A prática de exercícios aumenta a resistência dos ossos; retarda o processo de osteoporose; aumenta o tônus muscular; desenvolve a força e resistência muscular; preserva a saúde da coluna; protege as articulações de degenerações comumente encontradas entre os sedentários; proporciona maior desempenho físico no trabalho; proporciona maior tolerância à sobrecarga, propicia uma sensação de bem-estar geral; diminui a fadiga mental; diminui o nível de ansiedade; melhora o humor; melhora a qualidade do sono; gera maior integração social; desperta no grupo a necessidade da aplicação de princípios como espírito de equipe, método, disciplina e respeito ${ }^{16}$.

Saba ${ }^{17}$ alerta para o fato de que a motivação/sensibilização para um praticante manter-se constantemente em atividade física é o habito. Siqueira, Nunes e Zoboli ${ }^{18}$, em seu estudo concluíram que a prática da GL promove a médio e longo prazo um aumento significativo da flexibilidade, combatendo o encurtamento muscular e consequentemente reduzindo as dores musculares do cotidiano dos funcionários. Desta forma Figueiredo e Mont' Alvão ${ }^{19}$, quando se implanta um programa de GL numa empresa, envolve-se a coletividade, o que propicia, além dos benefícios físicos em si, momentos de descontração e um desligamento momentâneo dos problemas do trabalho, se tornando um momento de pausa.

A participação na GL incentiva a pratica de exercícios preventivos, atua combatendo vícios posturais promovem a socialização, consegue interferir em relação ao animo, 
integração, autoestima, autoconfiança e ate mesmo na autoimagem dos funcionários o que gera um bem estar físico e emocional, contribuindo na qualidade de vida dos mesmos, prevenindo doenças ocupacionais ${ }^{18}$. Segundo Polito e Bergamaschi ${ }^{2}$ a GL, além de prevenir a LER, busca a eficiência e saúde musculoesquelética, redução dos riscos ambientais, redução do estresse e interesse conjunto da empresa e dos funcionários em melhoria da qualidade de vida no trabalho.

Este trabalho tem como objetivo sensibilizar os funcionários que participam da $\mathrm{GL}$ a aderirem a alguma atividade física fora do ambiente de trabalho, analisar se eles após a participação nas aulas começaram a praticar alguma atividade física e em qual estagio de aderência encontram-se, além de verificar se houve alguma mudança entre os estágios de adesão a prática de $\mathrm{AF}$ (atividade física).

\section{METODOLOGIA}

O projeto foi aprovado pelo CEP com o protocolo 1309 e respeitados todos princípios éticos.

Realizou-se um trabalho visando conscientizar o publico alvo composto por 45 funcionários da Unoeste sobre como a ginástica pode levar eles a terem uma vida mais ativa. Além de um questionário que foi aplicado, houve um mural onde foi realizada a exposição de sugestões de atividades físicas e os benefícios desta prática, no intuito de sensibilizar e conscientizar a respeito de sua importância e após 20 dias o questionário foi novamente aplicado com o intuito de saber se houve ou não sensibilização de todos sobre a aderência a AF. O questionário contava com os seguintes questionamentos: 1- Eu não vou/ pré contemplação; 2- Eu talvez vá/ contemplação; 3Eu vou/ preparação; 4- Eu estou/ ação e 5- Eu tenho (... um habito saudável)/manutenção

\section{RESULTADOS}

Tabela - Logo abaixo estão às questões aplicadas aos funcionários e as porcentagens relativas às respostas encontradas:

\section{Questão 01.}

\begin{tabular}{l|lccc}
\hline & $\mathbf{1}$ & $\mathbf{2}$ & $\mathbf{3}$ & $\mathbf{4}$ \\
& $\mathrm{n}=(\%)$ & $\mathrm{n}$ (\%) & $\mathrm{n}$ ㅇ (\%) & $\mathrm{n}$ ㅇ (\%) \\
\hline $\begin{array}{l}\text { INFLUENCIA DA } \\
\text { GL NA SAÚDE }\end{array}$ & $0(0)$ & $0(0)$ & $4(8,8)$ & $41(91,2)$ \\
& & & & \\
\hline
\end{tabular}

A maioria dos funcionários sabe que a GL exerce certa influencia numa melhor qualidade de vida e em sua saúde, sendo assim podemos classificar este grupo dentro do estagio de Expectativa ou Contemplação, pois apesar de não terem o habito de praticar alguma Atividade Física, sabem da importância que ela tem. 


\section{Questão 02.}

\begin{tabular}{l|ccccc}
\hline & 1 & $\mathbf{2}$ & $\mathbf{3}$ & $\mathbf{4}$ & $\mathbf{5}$ \\
\hline & № (\%) & № (\%) & № (\%) & № (\%) & № (\%) \\
\hline $\begin{array}{l}\text { PRÁTICA DE } \\
\text { EXERCICIO }\end{array}$ & $1(2,2)$ & $3(6,6)$ & $6(13,3)$ & $14(31,1)$ & $21(46,6)$ \\
FISICO FORA & & & & & \\
DO TRABALHO & & & & & \\
& & & & & \\
\hline
\end{tabular}

A maior parte dos funcionários relatou não participar de nenhum tipo de Atividade Física fora de seu ambiente de trabalho, tendo como justificativa a falta de tempo, com isso, podemos então classificar este grupo dentro do estagio de Pré - contemplação ou Pré - expectativa, onde boa parte deles por serem sedentários não aderiu ou pretendem aderir a alguma $A F$, devido o pouco tempo que tem.

\section{Questão 03.}

\begin{tabular}{l|ccccc}
\hline \multicolumn{1}{c}{$\mathbf{1}$} & $\mathbf{2}$ & $\mathbf{3}$ & $\mathbf{4}$ & $\mathbf{5}$ \\
\hline & № (\%) & № (\%) & № (\%) & № (\%) & № (\%) \\
\hline $\begin{array}{l}\text { SITUAÇÃO ATUAL EM } \\
\text { RELAÇÃO À PRÁTICA } \\
\text { DE EXERCICIO FÍ́ICO }\end{array}$ & $2(4,4)$ & $17(37,7)$ & $19(42,2)$ & $4(8,8)$ & $3(6,6)$ \\
& & & & & \\
\hline
\end{tabular}

Observando que boa parte dos funcionários não tem o hábito de praticar Atividade Física, mas tem a consciência de que sua prática leva de encontro com uma melhor qualidade de vida, este grupo pode ser classificado no estagio de Expectativa ou Contemplação, pois sabem da sua importância, ou seja, apesar de não aderirem ainda a uma prática esportiva, pelo menos se conscientizam ao saber de sua importância.

\section{Questão 04.}

\begin{tabular}{l|cccc}
\hline \multicolumn{1}{c}{} & $\mathbf{1}$ & $\mathbf{2}$ & $\mathbf{3}$ & $\mathbf{4}$ \\
& № (\%) & № (\%) & № (\%) & № (\%) \\
\hline $\begin{array}{l}\text { A GL INFLUENCIOU } \\
\text { PARA A PRÁTICA DE }\end{array}$ & $2(4,4)$ & $1(2,2)$ & $9(20)$ & $33(73,3)$ \\
ATIVIDADE FÍSICA & & & & \\
& & & & \\
\hline
\end{tabular}

A maioria dos funcionários relatou que as aulas de GL dentro da empresa influenciaram decisivamente para que eles aderissem ou se conscientizassem da importância de praticar alguma AF, no seu dia-a-dia, com isso, podemos classificar este grupo dentro do estagio de Ação, onde boa parte dessas pessoas deram uma oportunidade de experimentar alguma AF, com isso é importante salientar que dentro deste período o educador físico tem um grande papel pois é 
nesta fase que as pessoas mais desistem, com isso é preciso motiva-los a dar continuidade nesta prática para que assim obtenham uma vida mais saudável e sem riscos de se lesionarem ou terem algum tipo de doença crônica.

\section{DISCUSSÃO}

Diversas situações que podem implicar no aparecimento de doenças, pois a inatividade física pode causar malefícios a nossa saúde, neste sentido, Nunomura ${ }^{20}$ divide em Quatro possibilidades os comportamentos frente à adoção da prática do exercício físico, sendo estas: Adoção: Crença dos benefícios proporcionados à saúde; é provável que a motivação esteja mais relacionada ao bem-estar; Manutenção: mais automotivados, estabelecendo as suas próprias metas, tem apoio familiar, a adesão está mais relacionada às sensações de bem estar e prazer, não percebem inconveniência nos exercícios; Desligamento: falta de tempo, e a inconveniência, falta de motivação, fatores situacionais, ocorre em maior número nos que tem histórico de inatividade ou baixas capacidades físicas e motoras; Retomada da atividade: melhor habilidade de administração do tempo, sensação de controle e autoconfiança, metas mais Flexíveis e pensamento positivo. Weinberg \& Gould $^{15}$ também descreveram aquilo que as pessoas apresentaram como desculpas para não se exercitar: falta de tempo: exames minuciosos revelam que esta seria uma questão de prioridade.

Cabe-se ressaltar que nosso estudo obteve êxito, onde percebemos que a grande maioria das pessoas apesar de não ser adeptas a alguma Atividade Física regular, tem conhecimento das mesmas e da sua contribuição na melhora da qualidade de vida.

\section{CONCLUSÃO}

Desta forma, concluímos que houve a sensibilização entre os funcionários, obtendo um índice elevado de subordinados alegando que pretende modificar suas atividades rotineiras adquirindo hábitos mais saudáveis e uma participação mais ativa durante as aulas de ginástica laboral.

\section{REFERÊNCIAS}

Carvalho SHF. Ginástica Laboral (Portal da saúde). Disponível em:<http:// w.w.w.df.trf1.gov.br/portalsaude>. Acesso em: 10-09-2004), 2004.

Polito E, Bergamaschi C. GL Teoria e Prática. 4ํe edição. Rio de Janeiro: Sprint, 2010, p.29.

Cantarino MR, Pinheiro EM. "Ginástica de pausa, trabalho e produtividade". Revista Brasileira de Educação Física e Desporto, Brasília. 1974;20:38-42.

Monteiro JC. Critérios utilizados na prevenção da L.E.R. Disponível em: < http:// eps.UFSC.br/disserta97/monteiro/cap3.htm> Acesso em: 19/07/99.

MGM. "Ginastica Laboral". Disponível em: <http:gold.com.br/ mgmprmc.htm> Acesso em: 22/05/99.

Bulsing SS. Ginástica Laboral (1998). Disponível em: <http:// w.w.w.pecanita.com.br/ginástica.htm>. Acesso em: 22 set. 1998.

Albuquerque, AMFC et al. Quem se beneficia dos programas de ginástica laboral. Cadernos de Psicologia social do trabalho. 2005;8:71-86. https://doi.org/10.11606/issn.1981-0490.v8i0p71-86 
Lima V. Ginástica laboral: atividade física no ambiente de trabalho. 3. ed. São Paulo: PHORTE, 2007.

Alter MJ. Ciência da flexibilidade. 2. ed. Porto Alegre: Artes Médicas, 1999.

Colegio Americano e Medicina do Esporte. Manual da Pesquisa do ACSM para os testes de esforço e sua prescrição. 4 ed. Rio de Janeiro: Guanabara Koogan, 2003.

Nahas MV. Atividade física, saúde e qualidade de vida: conceitos e sugestões para um estilo de vida ativo. 4. ed. Londrina: Midiograf, 2006.

Costa AF, Dias AG, Vale RGS. A contribuição de um programa de ginástica laboral para aderência ao exercício físico fora do ambiente de trabalho. 2006. Programa Stricto-sensu em Motricidade Humana - Universidade Castelo Branco. Rio de Janeiro.

Prochaska JO, Marcus BH. The transtheoretical model: applications to exercise. In DISHMAN, R. K (ed.) Advances in exercise Adherenci. Human. Kinetics, champaign, IL. 1994;161-180.

Reed GR, Velicer WF, Prochaska J0, Rossi JS \& Marcus BH. What makes a good staging algorithm: Examples from regular exercise. American Journal of Health Promotion.1997;12: 57-66. https://doi.org/10.4278/08901171-12.1.57.

Weinberg RS, Gould D. Fundamentos da psicologia do esporte e do exercício. 2. ed. Porto Alegre: Artmed, 2001.

Brandt Giordani L. GL e sua influência no estilo de vida: um estudo acerca de sua contribuição no relacionamento interpessoal, alimentação e pratica de atividade física. Trabalho de Conclusão de Curso (Graduação) -. Universidade Federal do Rio Grande do Sul, Escola de Educação Física. 2011:20-28.

Saba F. Aderência à prática do exercício físico em academia. São Paulo: Manole, 2001.

Soares Siqueira J, Cunha Nunes C, Zoboli F. A ginástica laboral e a qualidade de vida do trabalhador. 2011. Disponível em: <www.efdeportes.com>. Acesso em: 16 fev. 2012.

Figueiredo F, Montalvão C. Ginástica Laboral e Ergonomia. 2. ed. Rio de Janeiro: Sprint, 2008.

Nunomura M. Motivos de adesão à atividade física em função das variáveis idades, sexo, grau de instrução e tempo de permanência. Revista Brasileira de Atividade Física e Saúde. 1998;3(3):45- 